Title : will be set by the publisher

Editors : will be set by the publisher

EAS Publications Series, Vol. ?, 2018

\title{
CHEMODYNAMICS OF LYMAN ALPHA EMITTERS, LYMAN BREAK GALAXIES AND ELLIPTICAL GALAXIES
}

\author{
Masao Mori ${ }^{1}$ and Masayuki Umemura ${ }^{2}$
}

\begin{abstract}
We report an ultra-high-resolution simulation that follows evolution from the earliest stages of galaxy formation through the period of dynamical relaxation. The bubble structures of gas revealed in our simulation $\left(<3 \times 10^{8}\right.$ years $)$ resemble closely the high-redshift Lyman $\alpha$ emitters (LAEs). After $10^{9}$ years these bodies are dominated by stellar continuum radiation and look like the Lyman break galaxies (LBGs) known as the high-redshift star-forming galaxies at which point the abundance of elements appears to be solar. After $1.3 \times 10^{10}$ years, these galaxies resemble present-day ellipticals. The comparisons of simulation results with the observations of elliptical galaxies allow us to conclude that LAEs and LBGs are infants of elliptical galaxies or bulge systems in the nearby universe.
\end{abstract}

\section{Introduction}

Recent progress in observational devices and techniques has enhanced our knowledge of formation and evolution of galaxies on a firm statistical basis. Optical observations have revealed the presence of a number of LAEs at redshifts of $z \geq$ 3 (Dey et al. 1998; Taniguchi et al. 2003 and references therein) as well as LBGs at redshifts of $3 \leq z \leq 6$ (Steidel et al. 1996; Giavalisco 2002 and references therein). A good fraction of LBGs exhibit Ly $\alpha$ emission lines strong enough to be detected by narrow band observations (Shapley et al. 2003). Since LAEs and LBGs are quite young, they could hold direct interpretable information on the early chemical enrichment of galaxies, contrary to present-day galaxies which have undergone intense interstellar medium (ISM) recycling, thus erasing most of the early chemical history.

The modern paradigm of the galaxy formation, based on the cold dark matter hypothesis, deduces that galaxies formed hierarchically in a bottom-up fashion,

\footnotetext{
${ }^{1}$ Institute of Natural Sciences, Senshu University, Kawasaki, Kanagawa 214-8580, Japan

2 Center for Computational Sciences, University of Tsukuba, Tsukuba, Ibaraki 305-8577, Japan
} 
Title : will be set by the publisher

where a larger system results from the assembly of smaller dark matter halos. Baryonic gas falls into the gravitational potential of dark matter halos, and condenses rapidly as a result of the radiative cooling for atoms or molecules. The dense cooled gas clouds are the birth sites of stars, and massive stars born there explode as type II supernovae (SNe) in a few times $10^{7}$ years. Continual multiple SNe generate hot bubbles enriched with heavy elements and shocked dense shells. Hence, to study the chemical enrichment, it is crucial to resolve accurately the thermalization of the kinetic energy released by multiple SNe and the mixing of heavy elements. So far, the theoretical models of galactic chemical evolution have often assumed the homogeneous ISM (one-zone model), with the instantaneous and perfect mixing of heavy elements synthesized in SNe. However, the energy input and metal ejection by $\mathrm{SNe}$ are likely to proceed in an inhomogeneous fashion (Mori et al. 2004). Thus, simulations that can resolve SN remnants are required to properly model the chemical evolution of primordial galaxies. In this paper, we perform ultra-high resolution hydrodynamic simulations of a very large burst of multiple SN explosions in a forming galaxy. Also, by incorporating spectrophotometric modeling with the simulations, the results can be directly compared to the observations on high-redshift forming galaxies. The outline of this paper is as follows. In Section 2, the numerical method is described. In Section 3, we present the simulation results and in Section 4 the possible link among LAEs, LBGs and elliptical galaxies is discussed. The full description of this study can be found in Mori \& Umemura (2006).

\section{Numerical method}

Our simulation uses a hybrid $N$-body/hydrodynamics code which is applicable to a complex system consisting of dark matter, stars and gas. The gas is allowed to form stars and is subject to physical processes such as the radiative cooling and the energy feedback from SNe. The gas is assumed to be optically thin and in collisional-ionization equilibrium. Radiative cooling is included self-consistently with metallicity, using the metallicity-dependent cooling curves by Sutherland \& Dopita (1993). The collisionless dynamics for dark matter particles and stars is treated by the $N$-body method and the gas dynamics is pursued by a threedimensional AUSM-DV scheme that can treat shocks with high accuracy (Mori et al. 2002). Since this scheme has a great advantage due to the reduction of numerical viscosity, fluid interfaces are sharply preserved and small-scale features can be resolved.

Stars are assumed to form in rapidly cooling and Jeans unstable regions at a rate which is inversely proportional to the local dynamical time (see Mori et al. 1997, 1999). When a star particle is formed, we identify this with approximately

$10^{4}$ single stars and distribute the associated mass of the star particle over the single stars according to Salpeter's initial mass function. The lower and upper mass limits are taken as 0.1 and $100 M_{\odot}$, respectively. When a star particle is formed and identified with a stellar assemblage as described above, stars more massive than $8 M_{\odot}$ start to explode as Type II SNe with the explosion energy 
of $10^{51} \mathrm{ergs}$ and their outer layers are blown out with synthesized metals leaving the remnant of $1.4 M_{\odot}$. Consequently, once a new star particle is formed, the energy, metals and material from Type II SNe are subsequently supplied to 8 cells surrounding SN region. We compute the chemical evolution using the calculations of stellar nucleosynthesis products by Tsujimoto et al. (1995). A mass of $2.4 M_{\odot}$ of oxygen is ejected from a Type II SN explosion.

\section{Simulation}

Following a standard cold dark matter cosmology with cosmological constant $(\Lambda \mathrm{CDM})$, where we assume $\Omega_{M}=0.3, \Omega_{\Lambda}=0.7, \Omega_{b}=0.04$, and a Hubble constant of $H_{0}=70 \mathrm{~km} \mathrm{~s}^{-1} \mathrm{Mpc}^{-1}$, we consider the dynamical and chemical evolution of a protogalaxy with the total mass of $10^{11} M_{\odot}$. We assumed that the total mass of gaseous matter is $1.3 \times 10^{10} M_{\odot}$ initially. If we suppose a $2 \sigma$ density fluctuation, it decouples from the cosmic expansion and begins to contract at redshift $z=7.8$ with the radius of $53.7 \mathrm{kpc}$. The angular momentum is provided by a uniform rotation characterized by a spin parameter of $\lambda=0.05$. Prior to this galaxy-scale fluctuation, subgalactic dark halos collapse and are virialized. According to the $\Lambda \mathrm{CDM}$ cosmology, twenty subgalactic condensations with mass of $5.0 \times 10^{9} M_{\odot}$ and radius of $8.6 \mathrm{kpc}$ are distributed within the galaxy-scale fluctuation. The subgalactic virialized halos are assumed to follow the Navarro-Frenk-White density profile (Navarro et al. 1997). The hydrodynamic processes are pursued with $1024^{3}$ grid points. The simulation box has a physical size of $134 \mathrm{kpc}$ and the spatial resolution is $0.131 \mathrm{kpc}$.

Fig. 1 shows the result for the time sequence of the chemical enrichment, where the distributions of the logarithmic density, the temperature and the velocity, and oxygen abundance $[\mathrm{O} / \mathrm{H}]$, are presented until $10^{9}$ years. In the first $10^{8}$ years, stars are formed in high-density peaks within subgalactic condensations and the burst of star formation starts. Then, massive stars in the star forming regions explode as $\mathrm{SNe}$ one after another, producing expanding hot bubbles surrounded by cooled dense shells. The gas in the vicinity of SNe is quickly enriched with ejected heavy elements, but a large amount of gas still retains low heavy-element abundance. Consequently, the metallicity distribution becomes highly inhomogeneous, where gas enriched as $-5 \leq[O / H] \leq-1$ coexists with virtually primordial gas. Since the density of the ISM is lower in the outer regions of subgalactic condensations, the expansion of hot bubbles is accelerated there and SN-driven shocks collide with each other to generate super-bubbles of $\sim 50 \mathrm{kpc}$, and the surrounding highdensity, cooled $\left(T=10^{4} \mathrm{~K}\right)$ shells form at $3 \times 10^{8}$ years. The hot bubbles expand further by subsequent SN explosions, and the shells sweep up the partially enriched ambient gas. The gas density in dense shells increases owing to the efficient radiative cooling mainly through collisional excitation of neutral hydrogen.

After $5 \times 10^{8}$ years, the hot bubbles blow out into the intergalactic space. This $\mathrm{SN}$-driven outflow is an efficient mechanism to enrich the intergalactic medium with heavy elements over a large cosmological volume (see Mori et al. 2002). On the other hand, the dense shells undergoes hydrodynamic instabilities induced by 
Title : will be set by the publisher

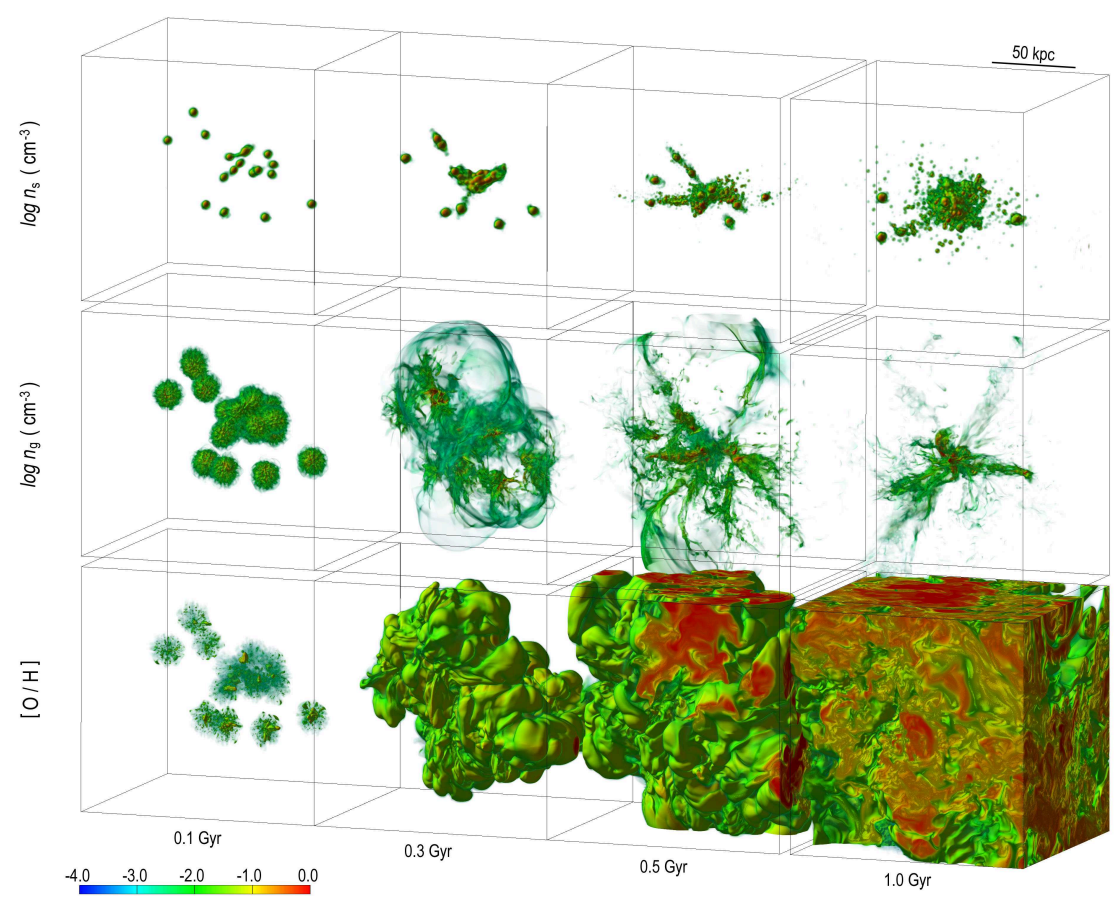

Fig. 1. Spatial distributions of the stellar density, the gas density, and the oxygen abundance $[\mathrm{O} / \mathrm{H}]$ of gas. The four panels in each column depict the time evolution of the simulation results until 1 Gyr.

shell-shell interactions and radiative cooling, eventually fragmenting into cold filaments and blobs. These interactions are giving rise to an intricate multiphase structure in the inner halo, where $10^{6-7} \mathrm{~K}$ gas coexists with a cooler $10^{4} \mathrm{~K}$ phase from which it is separated by cooling interfaces. New stars are born in this enriched gas and again heavy elements are ejected from subsequent SNe. The rightmost panels show the structure at $10^{9}$ years. By this stage, the ISM is recycled repeatedly and about $72 \%$ of the initial gas has been processed into stars. Eventually, some amounts of cool, dense filaments are left at the center. But, the most of volume is filled with rarefied gas $\left(n \leq 10^{-4} \mathrm{~cm}^{-3}\right)$ that has intermediate temperature $\left(10^{4.5} \mathrm{~K} \leq T \leq 10^{6.5} \mathrm{~K}\right)$. At this epoch, the mixing of heavy elements is nearly completed.

\section{Discussion and conclusion}

The emission properties of the gas components are calculated for an opticallythin, collisionally-ionized gas using MAPPINGIII code by Sutherland \& Dopita (1993), and those of the stellar components are calculated using the evolutionary 


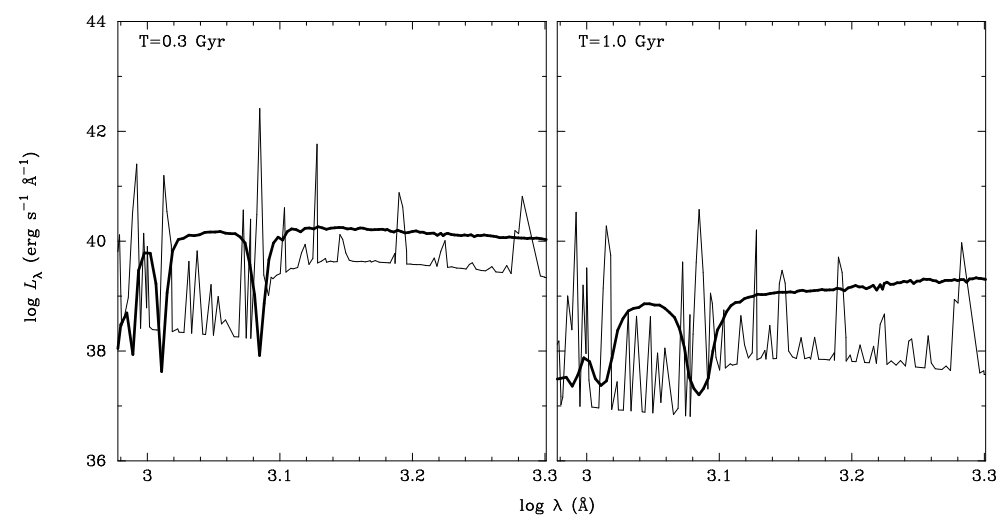

Fig. 2. Predicted emission properties of the gas components assuming for an optically thin gas in collisional-ionization equilibrium (thin lines), and that of the stellar components using the evolutionary stellar population synthesis (thick lines).

stellar population synthesis code PEGASE by Fioc \& Rocca-Volmerange (1997). In practice, to obtain the SED, we sum up the SED of each grid point for the gas components and each star particle for the stellar components. Thus, the present simulations can be directly compared to the observations.

Fig. 2 shows the spectral energy distribution (SED) of the simulated galaxy. Thin lines and thick lines depict, respectively, the gas emission and the stellar emission. The Ly $\alpha$ emission comes mainly from high-density cooling shells, and its luminosity is more than $10^{43} \mathrm{erg} \mathrm{s}^{-1}$ in the first $3 \times 10^{8}$ years. This Ly $\alpha$ luminosity perfectly matches that of observed LAEs (Matsuda et al. 2004). Furthermore, we find that the physical extent of $\sim 100 \mathrm{kpc}$ and the bubbly structure produced by multiple SNe are quite similar to the observed features in Ly $\alpha$ surface brightness distribution of LAEs. After $3 \times 10^{8}$ years, the Ly $\alpha$ luminosity quickly declines to several $10^{41} \mathrm{erg} \mathrm{s}^{-1}$ that is lower than the observed level. This result suggests that LAEs can correspond to an early SN-dominated phase before $3 \times 10^{8}$ years.

As seen in Fig. 2, after the sparkling phase of a primeval galaxy, the SED is dominated by stellar continuum emission, since the emission from cooling gas decreases immediately owing to the leak of explosion energy through the blowout of super-bubbles. The galaxy in this phase is featured with diffuse, asymmetric structures, and outflows of $100 \sim 500 \mathrm{~km} \mathrm{~s}^{-1}$. The total mass of long-lived stars is $9.3 \times 10^{9} M_{\odot}$, and the mass of $1.5 \times 10^{9} M_{\odot}$ is involved in the outflows at $z=3$. These features look quite similar to those observed for LBGs (Adelberger et al. 2003). In the light of such properties, the simulated post-starburst galaxy that has the age of $10^{9}$ years can correspond to LBGs.

The following dynamical evolution is studied by an $N$-body simulation with one million particles, to explore the end-product of this galaxy. As a result, it is found that the assembly of sub-condensations and the virialization of the total system 
Title : will be set by the publisher

are almost completed in $3 \times 10^{9}$ years, so that the system becomes in a quasiequilibrium state. The resultant stellar system forms a spherical virialized system. The projected surface brightness distributions have a large central concentration that well accords with de Vaucouleurs' $r^{1 / 4}$ profile and the resultant absolute magnitude in blue band and visual band are $M_{B}=-17.2 \mathrm{mag}$ and $M_{V}=-18.0$ mag, respectively. The colour $U-V=1.15$ and $V-K=2.85$ are consistent with the colour-magnitude relation of elliptical galaxies in Coma cluster of galaxies (Bower et al. 1992). Furthermore, the combination of the surface brightness, the effective radius $r_{e}=3.97 \mathrm{kpc}$, and the central velocity dispersion $\sigma_{0}=133 \mathrm{~km} \mathrm{~s}{ }^{-1}$ is on the fundamental plane of elliptical galaxies within their scatters (Djorgovski \& Davis 1987). These comparisons of the simulation results with the observations allow us to derive a significant conclusion that LAEs and LBGs are progenitors of present-day elliptical galaxies, and the on-going, major chemical enrichment phases.

\section{Acknowledgements}

This work was supported in part by the Grant-in-Aid of the JSPS, 14740132, and by Grants-in-Aid of the MEXT, 16002003. The computations reported here were performed on the Earth Simulator at the JAMSTEC, the SPACE at Senshu University, and the computational facilities at CCS in the University of Tsukuba.

\section{References}

Adelberger, K. L. et al. 2003, ApJ, 584, 45

Bower, R. G. et al. 1992, MNRAS, 254, 601

Dey, A. et al. 1998, ApJ, 498, L93

Djorgovski, S. \& Davis, M. 1987, ApJ, 313, 59

Fioc, M. \& Rocca-Volmerange, B. 1997, A\&A, 326, 950

Giavalisco, M. 2002, ARA\&A, 40, 579

Matsuda, Y. et al. 2004, AJ, 128, 569

Mori, M., Ferrara, A. \& Madau, P. 2002, ApJ, 571, 40

Mori, M. \& Umemura, M. 2006, Nature, 440, 644

Mori, M., Umemura, M. \& Ferrara, A. 2004, ApJ, 613, L97

Mori, M., Yoshii, Y. \& Nomoto, K. 1999, ApJ, 511, 585

Mori, M., Yoshii, Y., Tsujimoto, T. \& Nomoto, K. 1997, ApJ, 478, L21

Navarro, J. F., Frenk, C. S. \& White, S. D. M. 1997, ApJ, 490, 493

Shapley, A. E. et al. 2003, ApJ, 588, 65

Steidel, C. C. et al. 1996, AJ, 112, 352

Sutherland, R. S. \& Dopita, M. A. 1993, ApJS, 88, 253

Tanighichi, Y. et al. 2003, JKAS, 36, 123

Tsujimoto, T. et al. 1995, MNRAS, 277, 945 